\title{
Ambient Assisted Living: A Research on Human Activity Recognition and Vital Health Sign Monitoring using Deep Learning Approaches
}

\author{
Manoj T, Thyagaraju G S
}

\begin{abstract}
The rise in life expectancy rate and dwindled birth rate in new age society has led to the phenomenon of population ageing which is being witnessed across the world from past few decades. India is also a part of this demographic transition which will have the direct impact on the societal and economic conditions of the country. In order to effectively deal with the prevailing phenomenon, stakeholders involved are coming up with the Information and Communication Technology (ICT) based ecosystem to address the needs of elderly people such as independent living, activity recognition, vital health sign monitoring, prevention from social isolation etc. Ambient Assisted Living (AAL) is one such ecosystem which is capable of providing safe and secured living environment for the elderly and disabled people. In this paper we will focus on reviewing the sensor based Human Activity Recognition (HAR) and Vital Health Sign Monitoring (VHSM) which is applicable for $A A L$ environments. At first we generally describe the $A A L$ environment. Next we present brief insights into sensor modalities and different deep learning architectures. Later, we survey the existing literature for HAR and VHSM based on sensor modality and deep learning approach used.
\end{abstract}

Keywords - Ambient Assisted Living; Deep Learning; Human Activity Recognition; Vital Health Sign Monitoring

\section{INTRODUCTION}

The global phenomenon of population ageing among human population is seen more often in most of the countries across the world due to gradual decline in the birth and mortality rate. In years to come this demographic change will have serious implications on our society. The pace of population ageing has increased dramatically in most of the developed countries and some of them even attained the older population age structure than ever seen and heard in the past[1]. According to Department of Economic and Social Affairs, United Nations there about around 13\% of the global population comprising an estimated 962 million in the world are aged 60 or over in the world. The population aged 60 or above is increasing at a rate of about $3 \%$ per year [2].

In India, there are around 104 million people aged 60 or above which comprises the $8.6 \%$ of total population as per demographic reports. The ageing rate has increased over time from 5.6\% in 1961 and it is projected to grow around $20 \%$ of population by 2050 (i.e., around 34 million)[3]. This

Revised Version Manuscript Received on April 12, 2019.

Manoj T, Computer Science Engineering, Shri Madhwa Vadiraja Institute of Technology and Management, Bantakal, Udupi, Karnataka, India

Dr.Thyagaraju G S, Computer Science Engineering, SDM Institute of Technology, Ujire, Dakshina Kannada, Karnataka, India stark reality of spike in elderly population will be accompanied by surge in age-related chronic disorders such as dementia and other mental health issues, diabetes, cancer, cardio-vascular diseases, respiratory related issues etc., as well as with physical or cognitive impairments such as sensory loss, motor handicap, hyperactivity disorder etc. In order to alleviate the predicament of such aged or disabled population to be dependent on their family members or caregivers; a technological ecosystem which provides independent and healthy lifestyle is the need of the hour. Ambient Assisted Living (AAL) tries to fill this gap by making targeted interventions at various stages of assisting the elderly population. In recent years, various research communities, focus groups, businesses and policy makers have shown keen interest to develop a low-cost, reliable, secure, robust, eco-friendly assistive healthcare solutions to effectively counter the repercussions of aging population. However, gathering the ambient intelligence from the operational surroundings demands the confluence of multiple assistive techniques. It involves the scenarios such as human activity recognition, vital health sign monitoring, mobility assistance, gait analysis, sensor data analysis etc. The state of art deep learning approaches will leverage the experience of assisted living which facilitates in acquiring the ambient intelligence from the devices deployed in the AAL ecosystem.

This paper is organized into sections as follows: Section II provides the introduction to Ambient Assisted Living environment. Section III briefly summarizes sensor modality most commonly seen in sensor based monitoring Section IV describes the classification of deep learning architectures which is extensively used in the research work and many applications. Section V and VI respectively focuses on the critical review of HAR and VHS based on sensor modality and deep learning approach used. Section VII concludes the paper by discussing some of recent happenings related to research of HAR and VHSM using deep learning in AAL environment.

\section{AMBIENT ASSISTED LIVING}

The term of AAL has opened up new avenue to innovate cutting edge technologies to provide assistance as well as healthcare and rehabilitation to elderly people with physical or mental impairment

[4].

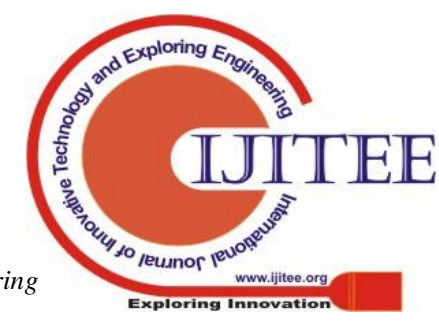


AAL is an interdisciplinary field which aims at exploiting information and communication technologies in smart and assistive healthcare to effectively counter the effect of spiraling elderly and disabled population [5]. In order to provide the ecosystem which delivers the quality health services and provide an independent living of their preference to the aged people, assistive technologies research and projects were initially conceived as Ambient Assisted Living in the year 2008 by European Framework Program. In the year 2014, the name of the programme has been changed into Active and Assisted Living for the second phase of research funding.

As the AAL solutions are being integrated into older adult's natural environment utmost care must be taken by developers in understanding the needs of end users and their care givers. In order to accomplish this, AAL programme has come up with precise of objectives as follows:

i. Be intuitively used by the elderly people, their dependents, formal and informal care givers.

ii. To increase the autonomy, self-confidence and mobility of elderly population to help them to stay independently.

iii. To extend the healthcare services for early detection of risks involved in aged related chronic disorders.

iv. To offer better and healthier lifestyle to elderly individuals and prevent them from social isolation.

v. To leverage the efficiency and productivity of ICT based resources in AAL environment.

In order to accomplish AAL tasks the ecosystem has to synergize the use of multiple cutting edge technological domains such as Context-aware computing, Sensor Networks, Ubiquitous Computing, Internet of Things, Machine Learning, Artificial Intelligence, Cognitive computing etc.

\section{SENSOR MODALITY}

In AAL based environment, myriad of sensors are integrated into the devices/objects which are intelligently interconnected by various network connectivity mechanisms. These sensors in turn generate lot of data which provides rich contextual information for activity recognition and vital sign monitoring. According [6] to the sensor based approaches adopted for HAR and VHSM most commonly make use of data modalities which can be categorized into three aspects: wearable/body-worn sensors, ambient sensors and hybrid sensors.

\section{A. Wearable/Body-Worn Sensors}

They are the class of sensors which can be directly worn on the body, implanted under skin or indirectly carried with the assistance of individual's body. They can be attached to mobile devices, smart phones, clothing, belt, shoes, wristwatches, headbands, glasses or even implanted in the body. Accelerometer along with gyroscope and magnetometer are mostly frequently used for activity recognition. They are most commonly seen in human embedded chips to monitor vital health signs such as body temperature, blood pressure, pulse rate, respiration rate, blood glucose level etc. Based on this sensor modality many researchers have proposed the deep learning approaches for HAR and VHSM.

\section{B. Ambient Sensors}

They are the class of sensors used to capture interaction between the operational vicinity and humans. The term "ambient sensors" stands for the intelligent goal specific sensors embedded in various devices or objects used in living environment which will interact with humans or other objects of similar type. They include various sensors such as passive infrared sensors (PIR), video sensors, pressure sensors, acoustic sensors, floor sensors, radar sensors etc. Several research works are being carried out in smart environment using ambient sensors which make use of deep learning techniques to recognize daily activities and monitor vital health signs. The deployment of ambient sensors is relatively difficult and it is measurements may be easily effected by the external environmental factors.

\section{Hybrid Sensors}

They are the class of sensors in which different types of sensors from both wearable and ambient sensors can be combined to accomplish a common task. Here the combination of sensors complements each other to overcome their limitations in an operating environment. For instance, value of accelerometer sensor can be combined with acoustic information from ambient sensor to improve the accuracy in activity recognition [7]. It is obvious that the combination of sensors is captures rich contextual information which can be effectively used by deep learning techniques in AAL scenarios.

\section{DEEP LEARNING ARCHITECTURES}

Deep learning, a subset of machine learning which models the several levels of features has become a trend in the area of image recognition, activity recognition, speech processing, natural language processing etc. The multiple layers of neural networks comprises of varied features represented hierarchically from lower to higher level. According to [8] deep learning architectures can be broadly classified into three categories: generative deep architecture, discriminative deep architecture and hybrid deep architecture.

\section{A. Generative Deep Architecture}

It intends to build the model by characterizing the higher-order correlation properties as well as joint distributions of the visible statistical data and their associated classes. The unsupervised feature learning is the characteristic of generative deep architectures since the labels for the data are not considered. Without relying upon on all layers above it is required to learn from lower layer in a greedy manner in a bottom up fashion. There are several deep models under this architecture but we discuss most prominent among them such as Recurrent Neural Network (RNN), Deep Belief Network (DBN), Restricted Boltzmann Machine (RBM),

Autoencoder etc. 


\section{Recurrent Neural Network}

They are the class of neural networks specialized for processing the sequential data. They are extensively used in the area of natural language processing, speech synthesis, image captioning, music generation, time series data processing etc. RNNs are capable of remembering inputs with the help of Long Short Term Memory (LSTM) units. In a conventional network, the assumption is that inputs and outputs are independent of each other but in case of RNNs they perform the similar task for each element of a sequence, with the output relying upon the previous computations. When they are used to model the sequential data it can be treated as the member of deep generative architecture [9].

\section{Deep Belief Network}

They are the class of probabilistic generative models comprising of multiple tiers of stochastic, latent variables. DBNs are composed of multiple tiers of hidden units where each tier is connected with another with no units connected within each tier. The undirected symmetric connections between the top two tiers form an associative memory. The lower layers receive top-down, directed connections from the layer above. DBNs learn through the values of latent variables by taking one layer into consideration when they are being inferred from data. DBN encompasses many simple learning modules which in turn is made up of restricted type of Boltzmann machine [10].

\section{Restricted Boltzmann Machine}

They are the class of fully connected, bipartite, undirected graphical model comprising of a hidden layer and visible layer [11].The hidden layer bias helps in providing the activation function during forward pass while the visible layer bias helps the RBMs to learn the reconstruction on the backward pass. RBMs are useful for accomplishing tasks such as classification, regression, dimensionality reduction, topic modeling and feature learning. They are shallow, two layered neural networks which forms the basis for DBNs and belongs to the category of Energy Based models. They represent a probability distribution which is parameterized generative models.

\section{Autoenconder}

They are the class of neural networks which are trained to attempt to copy its input to the output. They provide the smaller representation by reducing the size of the input and one can retrieve the original data by reconstructing the compressed data. They are most commonly used in applications such as Image Coloring, Feature Variation, Dimensionality Reduction, Watermark Removal, Denoising Image etc. Autoenconders uses the simple three layer representation which comprises of encoder, code, decoder. The input into the network is compressed into latent space representation by the encoder and the compressed code which is fed into the decoder is known as code. Finally, decoder is the one which decodes the compressed data into original dimension. Autoencoders are capable of compressing the data which they have seen earlier and decompressed outputs will be degraded compared to the original inputs. The different types of autoencoders which frequently used in research work are Sparse Autoencoders, Convolution Autoencoders, Deep Autoencoders, Contractive Autoenconders etc.

\section{B. Discriminative Deep Architecture}

It intends to build the model by conditioning the visible data on the posterior distributions of classes. A good amount of discriminative supervised learning techniques make use of shallow architectures such as Hidden Markov Models (HMMs) and Conditional Random Field (CRFs). But in present day research work various discriminative deep architectures such as Deep Fully Connected Networks (DFNs) and Convolutional Neural Networks (CNNs) are widely used.

\section{Deep Fully Connected Network}

They are the class of neural networks derived from traditional Artificial Neural Networks (ANNs) with numerous (deep) layers involved in it rather than having very shallow layers. DFNs are competent enough to learn from vast amount of data with help of deep layers associated with it. DFNs more often are the part of other deep models. They are structure agnostic which makes DFNs applicable to a wide range of problem domains in general but tend to have a weaker performance in comparison with special purpose networks which are tuned to the structure of a problem space. Besides that, there is no need to make special assumptions about the input.

\section{Convolutional Neural Networks}

They are the class of neural networks which comprises of neurons capable of learning weights and biases which are most extensively used for processing an image. CNNs include basic building blocks such as convolution layers, pooling layers and fully connected layers which are designed to adaptively and automatically learn multi-scale hierarchical spatial features from low level to high level patterns through backpropagation. They are the mathematical constructs comprising of convolution, pooling and fully connected layers. The feature extraction is done by the convolution and pooling layers respectively whereas the extracted features are mapped into final output by the third layer named fully connected layers [12]. CNNs are widely applicable in the area of object detection, image recognition, object identification, pose estimation, action recognition, text detection and recognition, scene labeling etc. The lower layer feeds the next higher layer the complexity of extracted features increases with the hierarchy. The process of optimization is performed in order to minimize difference between actual labels and outputs through backpropagation and refining weights by gradient descent.

\section{Hybrid Deep Architecture}

It refers to the model which is the blend of both generative discriminative and model

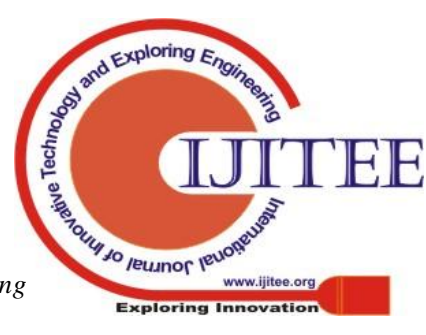


components. In this model, the generative component is augmented with the discriminative one which is the ultimate of hybrid architecture. The generative models can be the exceptional initialization points for highly non-linear parameter estimation problems and it controls the complexity of overall model from the regularization perspective.

\section{HUMAN ACTIVITY RECOGNITION}

The recognition of human activities in indoor and outdoor spaces is gaining profound attention in applications such as video based surveillance and security systems, personal fitness tracking, daily life monitoring, localization, ambient assisted living, industry manufacturing etc., which can be realized with greater proficiency with the rapid technological advancements in the field of Sensor technology, Computer Vision and Pattern Recognition, Machine Intelligence, Internet of Things, Context Awareness and Big Data Analytics. One of the most frequently encountered scenarios in the Ambient Assisted Living (AAL) is the Human Activity Recognition (HAR) of the aged seniors within their dwelling spaces. A HAR component performs the task of recognizing human activity patterns from various types of raw data collected from AAL devices. The conventional vision based approaches has yielded good amount of results for HAR in the past years. But the high dependence of these approaches on heuristic hand-crafted feature extraction methods degrades its generalization performance. In addition to that, it yields the less accurate results for unsupervised and incremental learning tasks. In order to overcome these limitations our review work focuses on sensor based HAR where cutting edge deep learning techniques can be applied.

\section{A. Sensor-Based Human Activity Recognition}

Almost all of the human activity recognition approaches

Table I: Sensor-based Human Activity Recognition using Generative Deep Learning Architecture

\begin{tabular}{|c|c|c|c|c|}
\hline Reference & Sensor Modality & $\begin{array}{c}\text { Deep Learning } \\
\text { Model }\end{array}$ & Description & Dataset \\
\hline Zhang et al.,2015[13] & $\begin{array}{c}\text { Body-Worn } \\
\text { (Accelerometer) }\end{array}$ & $\mathrm{DBN}$ & $\begin{array}{l}\text { Recommended a Deep Belief Network (DBN) for } \\
\text { real-time activity recognition where every two } \\
\text { adjacent layers are treated as Restricted Boltzmann } \\
\text { Machine(RBM) and are initialized using the } \\
\text { generative pre-training. }\end{array}$ & Self \\
\hline $\begin{array}{l}\text { Alsheikh et al., } \\
\text { 2016[14] }\end{array}$ & $\begin{array}{c}\text { Body-Worn } \\
\text { (Accelerometer) }\end{array}$ & $\mathrm{DBN}$ & $\begin{array}{c}\text { Developed a Deep Belief Network(DBN) based } \\
\text { model for human activity recognition considering } \\
\text { triaxial accelerometer data which comprised of } \\
\text { multiple layers of hidden units }\end{array}$ & $\begin{array}{c}\text { WISDM Actitracker, Daphnet } \\
\text { and Skoda }\end{array}$ \\
\hline $\begin{array}{c}\text { Bhattacharya et } \\
\text { al.,2016[15] }\end{array}$ & $\begin{array}{c}\text { Body-Worn } \\
\text { (Accelerometer) }\end{array}$ & $\mathrm{RBM}$ & $\begin{array}{l}\text { Application of Restricted Boltzmann Machine } \\
\text { (RBM) to perform variety of context-aware } \\
\text { recognition tasks such as mode of transport, physical } \\
\text { activities of an individual and location detection in } \\
\text { indoor/outdoor spaces. }\end{array}$ & Self, OPPORUNITY \\
\hline Wang et al.,2016[16] & Ambient Sensors & SAE & $\begin{array}{l}\text { Developed an integrated framework using stacked } \\
\text { autoencoder (SAE) for the activity recognition. The } \\
\text { weights and biases required for training the network } \\
\text { is obtained through backpropagation and optimized } \\
\text { using gradient descent algorithm. }\end{array}$ & $\begin{array}{c}\text { Public dataset from Intelligent } \\
\text { Systems Lab }\end{array}$ \\
\hline
\end{tabular}

involve various modalities of collecting the sensor data. Most common among them are body-worn/wearable sensors, ambient sensors and hybrid sensors. The body-worn sensors such as smartphones, watches, bands, helmets and glasses offer a rich source of data for activity recognition. The components such as accelerometer and gyroscope which are embedded in these body-worn sensors play a pivotal role in obtaining the requisite information for activity recognition. They are particularly suitable when there is a repetitive body motion such as walking, running, standing, sitting and climbing stairs etc. The location based activity recognition will play a prime role as aged people with independent living may change their location based on their requirements. In some specific circumstances the wearable sensors may not be widely deployed since it may be intrusive in nature or user is required to carry corresponding sensors all the time. For ex: In AAL environments which involves the multiple actors within the interaction space such as aged person's interaction with one or more living or non-living entities(shaking hands, giving a hug), multiple people involved in cooking, medication intake, dressing etc. However, in order to overcome this limitation ambient sensors can be used to recognize complex activities. These ambient sensors are widely deployed in AAL environment in order to augment the capability of wearable sensors in activity recognition. The proper mix of wearable as well as ambient sensors which are termed as hybrid sensors can improve accuracy and precision of activity recognition. Table I reviews various related research papers based on generative deep learning architecture for activity recognition Table II provides the review of some research papers based on discriminative deep learning architecture for HAR and Table III offers the provides review of hybrid deep leaning architecture used in activity recognition. using gradient descent algorithm. 


\begin{tabular}{|c|c|c|c}
\hline $\begin{array}{l}\text { Almaslukh et } \\
\text { al.,2017[17] }\end{array}$ & $\begin{array}{c}\text { Body-Worn } \\
\text { (Accelerometer and } \\
\text { Gyroscope) }\end{array}$ & SAE & $\begin{array}{c}\text { Proposed a cost-effective Stacked AutoEncoder } \\
\text { (SAE) framework for smartphone based activity } \\
\text { recognition. The parameters of estimation are } \\
\text { fine-tuned according to best practices followed } \\
\text { thereby increasing the accuracy of recognition tasks. }\end{array}$ \\
\hline
\end{tabular}

Table II: Sensor-based Human Activity Recognition using Discriminative Deep Learning Architecture

\begin{tabular}{|c|c|c|c|c|}
\hline Reference & Sensor Modality & $\begin{array}{c}\text { Deep Learning } \\
\text { Model }\end{array}$ & Description & Dataset \\
\hline Zeng et al.,2014[18] & $\begin{array}{c}\text { Body-Worn } \\
\text { (Accelerometer) }\end{array}$ & $\mathrm{CNN}$ & $\begin{array}{l}\text { Developed a method based on Convolutional Neural } \\
\text { Networks (CNN) for activity recognition in which } \\
\text { features extracted based on local dependency. }\end{array}$ & $\begin{array}{l}\text { OPPORTUNITY, Skoda and } \\
\text { Actitracker(public dataset) }\end{array}$ \\
\hline Jiang et al., 2015[19] & $\begin{array}{l}\text { Body-Worn } \\
\text { (Accelerometer and } \\
\text { Gyroscope) }\end{array}$ & $\begin{array}{c}\mathrm{CNN} \text { and CNN } \\
\text { with weight sharing }\end{array}$ & $\begin{array}{l}\text { Proposed an approach of assembling the signal } \\
\text { sequences from accelerometer and gyroscope into a } \\
\text { novel activity image. Later, a Deep Convolutional } \\
\text { Neural Networks (DCNN) model is used to } \\
\text { recognize the activity from the image. }\end{array}$ & $\begin{array}{l}\text { UCI smartphone, } \\
\text { USC-HAD,SHO }\end{array}$ \\
\hline Inoue et al.,2016[20] & $\begin{array}{c}\text { Body Worn } \\
\text { (Accelerometer) }\end{array}$ & RNN & $\begin{array}{l}\text { Proposed a method of human activity recognition by } \\
\text { applying deep recurrent neural network (DRNN) on } \\
\text { raw accelerometer data. The various combinations } \\
\text { of architectures were investigated for the better } \\
\text { estimation of parameter values. }\end{array}$ & HASC open dataset \\
\hline $\begin{array}{c}\text { Edel \& } \\
\text { Koppe,2016[21] }\end{array}$ & $\begin{array}{c}\text { Body Worn } \\
\text { (Accelerometer and } \\
\text { Gyroscope) }\end{array}$ & RNN & $\begin{array}{l}\text { Developed optimized binary version of Bidirectional } \\
\text { LSTM (Long Short Term Memory) along with } \\
\text { RNN for human activity recognition }\end{array}$ & OPPORTUNITY and PAMAP2 \\
\hline Ravi et al., 2017[22] & $\begin{array}{c}\text { Body-Worn } \\
\text { (Accelerometer and } \\
\text { Gyroscope) }\end{array}$ & $\mathrm{CNN}$ & $\begin{array}{l}\text { Proposed a deep learning methodology, which } \\
\text { combines the raw data obtained from accelerometer } \\
\text { and gyroscope together with additional information } \\
\text { derived from a set of shallow features in order to } \\
\text { increase accuracy of activity recognition. }\end{array}$ & $\begin{array}{l}\text { ActiveMiles smartphone, } \\
\text { WISDM v1.1 smartphone, } \\
\text { WISDM v2.0,Daphnet FoG }\end{array}$ \\
\hline Lee et al., 2017[23] & $\begin{array}{c}\text { Body-Worn } \\
\text { (Accelerometer) }\end{array}$ & $\mathrm{CNN}$ & $\begin{array}{l}\text { Proposed a one-dimensional (1D) Convolutional } \\
\text { Neural Network (CNN) framework for recognizing } \\
\text { human activity using raw data collected from triaxial } \\
\text { accelerometer from user's smartphones. }\end{array}$ & Self \\
\hline
\end{tabular}

Table III: Sensor-based Human Activity Recognition using Hybrid Deep Learning Architecture

\begin{tabular}{|c|c|c|c|c|}
\hline Reference & Sensor Modality & $\begin{array}{c}\text { Deep Learning } \\
\text { Model }\end{array}$ & Description & Dataset \\
\hline Morales et al.,2016[24] & $\begin{array}{l}\text { Body-Worn } \\
\text { (Accelerometer and } \\
\text { Gyroscope) }\end{array}$ & CNN+ LSTM & $\begin{array}{l}\text { Developed a model which transfers the features } \\
\text { learned by the Convolution Neural Networks to the } \\
\text { Long Short Term Memory(LSTM) which } \\
\text { understands the temporal sequence for recognizing a } \\
\text { particular activity. }\end{array}$ & $\begin{array}{l}\text { OPPORUNITY and Skoda Mini } \\
\text { Checkpoint }\end{array}$ \\
\hline Zheng et al.,2016[25] & $\begin{array}{l}\text { Body-Worn } \\
\text { (Accelerometer and } \\
\text { Gyroscope) }\end{array}$ & $\mathrm{CNN}+\mathrm{SAE}$ & $\begin{array}{c}\text { Proposed an innovative deep learning framework } \\
\text { which combines the univariate sequential time series } \\
\text { data from single channel into multiple channels for } \\
\text { feature representation. }\end{array}$ & PAMAP2 \\
\hline $\begin{array}{c}\text { Guan \& } \\
\text { Ploetz,2017[26] }\end{array}$ & $\begin{array}{l}\text { Body-Worn } \\
\text { (Accelerometer and } \\
\text { Gyroscope) }\end{array}$ & RNN+LSTM & $\begin{array}{c}\text { Proposed an ensembles of deep Long Short Term } \\
\text { Memory (LSTM ) networks along with RNN for the } \\
\text { real-time human activity recognition using } \\
\text { wearables. }\end{array}$ & $\begin{array}{c}\text { OPPORUNITY, PAMAP2, and } \\
\text { Skoda }\end{array}$ \\
\hline Li et al.,2017[27] & $\begin{array}{l}\text { Ambient and } \\
\text { Object }\end{array}$ & $\mathrm{CNN}+\mathrm{RNN}$ & $\begin{array}{l}\text { Developed a model based on Convolution Neural } \\
\text { Networks along with Long Short Term Memory } \\
\text { (LSTM) which collects the spatial and temporal } \\
\text { features from the different types of sensors. The } \\
\text { collected features are merged in the second step for } \\
\text { making decision about the activities. }\end{array}$ & $\begin{array}{c}\text { Charades, Olympic Sports and } \\
\text { Trauma Resuscitation }\end{array}$ \\
\hline Alzantot.,2017[28] & $\begin{array}{l}\text { Body-Worn } \\
\text { (Accelerometer } \\
\text { and Gyroscope) }\end{array}$ & LSTM+MDN & $\begin{array}{c}\text { Developed a model for synthesizing the sensor data } \\
\text { based on generative model which is made up of } \\
\text { numerous Long Short Term Memory(LSTM) } \\
\text { networks. It is augmented by the discriminatory } \\
\text { model named Mixture Density Network(MDN) for } \\
\text { identifying difference between actual and } \\
\text { synthesized data. }\end{array}$ & Self \\
\hline
\end{tabular}

\section{VITAL HEALTH SIGN MONITORING \& RESULTS}

The smart devices/objects within an AAL ecosystem embedded with wearable and ambient sensors are transcending the barriers and transforming the elderly/patient health monitoring. The monitoring of vital health signs with the help of consumer wearable devices has now become the most prevalent scenario in our daily lives as the distinction between the consumer wearables and medical devices is gradually fading away. These wearables offer access to the analytics at personal level and facilitate

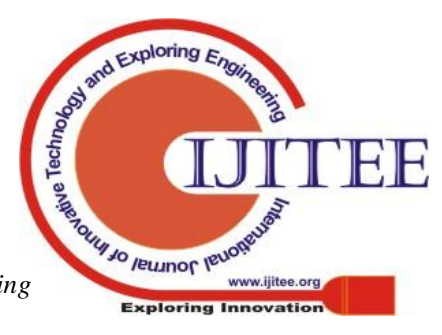




\section{Ambient Assisted Living: A Research On Human Activity Recognition And Vital Health Sign Monitoring Using Deep Learning Approaches}

the preventive care and help in managing prevailing illness [29]. The aged population ailing from the chronic disorders typically requires some kind of vital health signs monitoring especially in the advanced stages of disease progression. The chronic disorder monitoring involves the episodic patient monitoring which is utilized for the non-critical patients to track the vital health signs (body temperature, blood pressure, heart rate, respiration rate, heart rate, blood glucose) and identify the recovery or progress from the disorder; continuous patient monitoring deals with the acute conditions which requires continuous or frequent monitoring of vital signs; patient alarm monitoring encompasses triggering alarms based on preset conditions where person's vital signs and disorder specific indicators are monitored on a continuous basis[30]. In particular, the availability of non-intrusive and wearable sensors facilitates the episodic and continuous monitoring of human vital signs. Four major vital signs of human body are body temperature, heart rate, respiration rate and blood pressure [31]. In addition to that blood glucose level and pulse oxygenation are also widely used by healthcare professionals even though they are not the vital signs. Apart from vital signs many researchers have worked extensively on monitoring and analyzing the Electroencephalogram (EEG), Electromyography (EMG), sleep quality etc.

\section{A. Body Temperature}

A person's body temperature plays a pivotal role in the determining the physiological state. A normal body temperature for the adult in rest is around 37 degree Celsius. Due to variation in metabolism temperature fluctuates. For instance, the body temperature will be comparatively lower during the early morning since the metabolic rate will be slow for the body in resting state and it is relatively higher at the night after daytime due to food intake and physical activity. The body temperature varies based on the different locations (oral, core body, rectal) and the abnormal temperature at any of these locations is an indicator that a person may be suffering from a fever, infection etc. Temperature sensors can measure the amount of heat that is present in the body. Body temperature can be measured by using the thermoelectric effect, the thermistor or by optical means. Thermistor is the most widely used technique for non-intrusive and wearable temperature measurement. With the help of commercially available thermistor and temperature ICs which are known as contact temperature sensors, the measurement of temperature can be easily obtained. But these contact temperature sensors does not provide exact measurement of core body temperature which requires the calibration algorithm to get the exact value.

\section{B. Heart Rate}

Heart rate or pulse is undisputedly the most pivotal vital sign to determine the normal functioning of the human body. In order to consider a person to be healthy the heart must be in a perfect working condition. It is a measure of the number of beats per minute and useful for monitoring the cardiovascular activity of the heart. The primary responsibility of the human heart is to pump the oxygenated blood and nutrients to all parts of the body and remove waste from deoxygenated blood. A healthy adult human heart at rest will beat around 60-100 times per minute. A heart rate can be measured accurately using various approaches such as electrical, optical and strain sensors in comparison with manual measurement using stethoscope. The electrical measurement of heart rate involves techniques where electrocardiography (ECG) is measured using silver-coated chest suction electrodes without gel or paste and gold plated electrodes [32], a pair of epidermal electrodes in a band aid form factor [33]. A powerful approach called Photoplethysmography (PPG) makes use of optical and strain sensors to measure the heart rate. It is a very simple and low-cost optical technique which can be used to diagnose blood volume changes in the microvascular bed of tissue. It is one of the non-intrusive method to make measurements at skin surface [34].

\section{Respiration Rate}

The respiration (breathing) rate (RR) is the key physiological indicator to determine the health status of a person. The rate of respiration may change due to stress or illness. It is the total no of breathes or respiratory cycles that takes place per minute. Abnormal breathing rate is an indicator of disorders such as asthma, sleep apnea, obstructive pulmonary disorders which are caused due to insufficient oxygen inhalation and carbon dioxide exhalation from the body. A healthy resting adult will approximately have a one breath for every 6.4 seconds. Generally, the long-term RR monitoring can be categorized into direct and indirect approaches. The direct approach involves detecting the airflow during the breathing process and indirect approach involves monitoring and responding to chest and abdomen expansion and contraction during breathing. According to [35] the direct approach deploys the breath flow sensors near the nose or mouth that responds to fluctuations in air pressure, temperature, humidity, or carbon dioxide concentration as breathing takes place. The indirect method measures variations in physical parameters such as detecting the changes in lung volume or monitoring the chest area for activity. For ex: [36] designed wearable sensing system for long-term RR monitoring and [37] developed a respiration belt using weft-knitted strain sensors to monitor RR.

\section{Blood Pressure}

Blood pressure is the relevant health sign which measures the pressure of the blood within a circulatory system. The blood pressure is normally measured in terms of numbers known as systolic pressure (maxima- maximum during one heartbeat) and diastolic pressure (minima-minimum during one heartbeat) and expressed in millimeters of mercury $(\mathrm{mmHg})$. A healthy adult person will have approximately around $120 \mathrm{mmHg}$ systolic pressure and $80 \mathrm{mmHg}$ diastolic pressure which can be abbreviated as $120 / 80 \mathrm{mmHg}$. A steep increase (hypertension) or decrease (hypotension) in the blood pressure is an early 
indication for some kind of abnormality in human body. The blood pressure value above $140 / 90 \mathrm{mmHg}$ or below $120 / \mathrm{mmHg}$ is considered to be a matter of concern. A prolonged exposure to the hypertension will increase the chance of acquiring the ailments such as heart attack, stroke, renal disorders etc., and also hypotension may lead to conditions such as light-headedness, dizziness, faintness and falls. Even though the exact causes for fluctuations in blood pressure is still under the investigation, usually the stress, overweight, food intake with high salt and fat contents are potential reasons for variation in normal blood pressure. Traditionally, blood pressure is determined by sphygmomanometers. Due to its non-portable and static nature, it is not suitable to the AAL ecosystem which demands continuous measurement of vital health signs. According to [38], the present day sensor based blood pressure measuring systems utilize a capacitive sensitive strain sensor which encompasses both piezoelectric and compressible capacitive strain sensors.

\section{E. Blood Glucose}

Blood glucose level is one of the major human body parameter extensively used in determining the normal working condition of human body even though it is not included in the list of vital health signs. The human cells derive their energy from the glucose and it is carried to the entire body through means of blood stream. The amount of glucose present in the blood refers to the blood glucose level of the human body. According to American Diabetes Association (ADA), normal blood glucose level for a healthy adult should in a range $80-130 \mathrm{mg} / \mathrm{dl}$ before food intake and below $180 \mathrm{mg} / \mathrm{dl}$ one-two hours after food intake. If the blood glucose level is too high (hyperglycemia) too low (hypoglycemia) then it is detrimental to normal functioning of human body. Hyperglycemia may lead to complications such as frequent urination, increased thirst, excessive fatigue, nausea and vomiting, increased appetite and blurry vision and hypoglycemia is accompanied by conditions such as muddled thinking, sleep disturbances, mood swings, food aversion, poor balance, jitteriness etc. Therefore, continuous monitoring of blood glucose level is of utmost importance. Generally, the approaches for blood glucose measurement may be classified into invasive (which requires blood) and non-invasive (which does not required blood) [31]. The invasive method for continuous monitoring is inconvenient as it involves procedures such as obtaining blood for sampling, testing in the lab and then analyzing the results. As far as AAL environment is concerned non-invasive is the most appropriate method. In a non-invasive approach initial efforts were done based on electrochemical which used enzyme named glucose oxidase to determine the level of glucose [39]. The technique of measuring glucose level with the help of interstitial fluid is well known method which encompasses sensing techniques such as Reverse Iontophoresis, Thermal Emission, Photoacoustic Detection, Sonophoresis. Similarly the urine, sweat, breath, saliva, ocular fluid can be analyzed to determine glucose level in a non-invasive way [40]. Table IV reviews available literature where generative deep learning is applied for vital sign monitoring. Similarly, Table V and Table VI provide the review of research papers based on discriminative and hybrid deep architectures respectively.

Table IV: Sensor-based Vital Health Monitoring using Generative Deep Learning Architecture

\begin{tabular}{|c|c|c|c|c|}
\hline Reference & Sensor Modality & $\begin{array}{c}\text { Deep Learning } \\
\text { Model }\end{array}$ & Description & Dataset \\
\hline Jindal et al.,2016 [41] & $\begin{array}{c}\text { Body-Worn } \\
\text { (Pulse oximeter and } \\
\text { Accelerometer) }\end{array}$ & $\mathrm{DBN}+\mathrm{RBM}$ & $\begin{array}{c}\text { Proposed a novel approach for PPG-based } \\
\text { identification using two stage deep learning model } \\
\text { based on DBN and RBM. They used TROIKA } \\
\text { dataset for validation and achieved an accuracy of } \\
96.1 \%\end{array}$ & $\begin{array}{l}\text { Self and TROIKA as benchmark } \\
\text { dataset }\end{array}$ \\
\hline Lee et al.,2017[42] & $\begin{array}{l}\text { Body-Worn(Wrist } \\
\text { BP monitors) }\end{array}$ & $\mathrm{DBN}+\mathrm{DNN}$ & $\begin{array}{l}\text { Proposed a DBN-DNN regression model to estimate } \\
\text { the Blood Pressure(BP) by learning the complicated } \\
\text { non-linear relationship between feature vectors } \\
\text { extracted from Oscillometric Waveform's (OMW) } \\
\text { and target BPs. }\end{array}$ & $\begin{array}{c}\text { Self } \\
{[\text { Data collected from wrist BP }} \\
\text { monitors }]\end{array}$ \\
\hline Gu.,2017[43] & $\begin{array}{l}\text { Body-Worn(Blood } \\
\text { Glucose monitors) }\end{array}$ & RNN & $\begin{array}{l}\text { Developed a deep learning framework called } \\
\text { BGMonitor for non-invasive blood glucose } \\
\text { monitoring providing customized settings through } \\
\text { smartphone. The framework encompasses of three } \\
\text { major components such as external factor collection } \\
\text { module which collects the user related inputs such as } \\
\text { diabetes type, food intake, drug, insulin ; deepRNN } \\
\text { module is trained based on user inputs where } \\
\text { dynamic relationship between various contextual } \\
\text { parameters and blood glucose level; blood glucose } \\
\text { level tracking module determines glucose level } \\
\text { based on the results of previous module. }\end{array}$ & $\begin{array}{l}\text { Self } \\
\text { [Data collected from BG } \\
\text { monitor }]\end{array}$ \\
\hline Li et al., 2017[44] & $\begin{array}{c}\text { Ambient } \\
\text { (Wireless Home BP } \\
\text { monitor) }\end{array}$ & RNN with LSTM & $\begin{array}{l}\text { Proposed a novel RNN with LSTM model which is } \\
\text { capable of simultaneously combining the historical } \\
\text { measurement data (e.g. BP and heart rate) and } \\
\text { contextual data(e.g. age, gender, BMI) to predict the } \\
\text { early trends of user's blood pressure. }\end{array}$ & $\begin{array}{c}\text { Self } \\
\text { [Data collected from Wireless } \\
\text { home BP monitor] }\end{array}$ \\
\hline
\end{tabular}


Ambient Assisted Living: A Research On Human Activity Recognition And Vital Health Sign Monitoring Using Deep Learning Approaches

\begin{tabular}{|c|c|c|c|c|}
\hline Su et al.,2018[45] & $\begin{array}{l}\text { Body-Worn } \\
\text { (Biopac and } \\
\text { Finapres systems) }\end{array}$ & $\begin{array}{l}\text { RNN with } \\
\text { bidirectional } \\
\text { LSTM }\end{array}$ & $\begin{array}{l}\text { Proposed a novel approach based on RNN with } \\
\text { bidirectional LSTM for long term blood pressure } \\
\text { monitoring where the conventional approaches such } \\
\text { as regression models(decision tree, support vector } \\
\text { regression) and pulse-transit models fails in } \\
\text { achieving the required accuracy especially for } \\
\text { multi-day continuous BP prediction. They tested } \\
\text { their model for both static and multi day continuous } \\
\text { BP dataset }\end{array}$ & $\begin{array}{c}\text { Self } \\
\text { [Data collected using Biopac and } \\
\text { Finapres systems }]\end{array}$ \\
\hline $\begin{array}{l}\text { Martinsson et } \\
\text { al.,2018[46] }\end{array}$ & Multi-user input & RNN & $\begin{array}{l}\text { Proposed a method based on RNN for automatic } \\
\text { continuous blood glucose monitoring based on } \\
\text { historical data of blood glucose level of user's. This } \\
\text { method estimates the certainty of prediction and } \\
\text { helps user's to interpret the results. }\end{array}$ & Ohio T1DM dataset \\
\hline Sun et al.,2018[47] & Multi-user input & RNN with LSTM & $\begin{array}{l}\text { Proposed a RNN with one sequential bidirectional } \\
\text { LSTM layer and several fully connected layers to } \\
\text { predict the imminent hyperglycemia for diabetes } \\
\text { patients. }\end{array}$ & $\begin{array}{c}\text { Real-time patient and in silico } \\
\text { (UVa/Padova T1D Simulator) } \\
\text { datasets }\end{array}$ \\
\hline
\end{tabular}

Table V: Sensor-based Vital Health Monitoring using Discriminative Deep Learning Architecture

\begin{tabular}{|c|c|c|c|c|}
\hline Reference & Sensor Modality & $\begin{array}{c}\text { Deep Learning } \\
\text { Model }\end{array}$ & Description & Dataset \\
\hline Cho et al.,2017[48] & $\begin{array}{c}\text { Ambient } \\
\text { (Thermal Cameras) }\end{array}$ & $\mathrm{CNN}$ & $\begin{array}{l}\text { Deep Convolutional Neural Network(DCNN) based } \\
\text { deep learning model called DeepBreath was } \\
\text { proposed by them to automatically determine the } \\
\text { stress level of subjects(human being) based on } \\
\text { breathing patterns. The model introduced three fold } \\
\text { impact on learning by considering the spectrogram of } \\
\text { two dimensional respiration variability instead of } \\
\text { handcrafted features; application of CNN on } \\
\text { spectrogram and data augmentation technique to } \\
\text { learn with small-scale dataset. }\end{array}$ & $\begin{array}{c}\text { Self } \\
\text { [Data collected during Stroop } \\
\text { colour word test, Mental } \\
\text { computation test] }\end{array}$ \\
\hline Khan et al.,2017[49] & $\begin{array}{c}\text { Ambient } \\
\text { (WiFi sensing) }\end{array}$ & $\mathrm{CNN}$ & $\begin{array}{l}\text { Introduced a DCNN based end-to-end deep learning } \\
\text { framework for respiration monitoring using passive } \\
\text { WiFi sensing. They have combined CNN and } \\
\text { random forest estimator to determine the breathing } \\
\text { activity and breathing rate respectively. }\end{array}$ & $\begin{array}{l}\text { Self } \\
\text { [Data collected during WiFi } \\
\text { sensing] }\end{array}$ \\
\hline $\begin{array}{c}\text { Acharya et al., } \\
\text { 2017[50] }\end{array}$ & Body-Worn & $\mathrm{CNN}$ & $\begin{array}{l}\text { Proposed DCNN based novel approach for } \\
\text { automated detection of Coronary Artery } \\
\text { Disease(CAD) using variable durations of ECG } \\
\text { segments. The CNN model comprises of four } \\
\text { convolutional layers, four max pooling layers and } \\
\text { three fully connected layers along with the two and } \\
\text { five seconds duration of ECG signal segments. }\end{array}$ & $\begin{array}{c}\text { ECG Signals from Physionet } \\
\text { databases such as Fantasia(for } \\
\text { normal) and St.-Petersburg } \\
\text { Institute of Cardiology Technics } \\
\text { 12- lead arrhythmia(for CAD) }\end{array}$ \\
\hline $\begin{array}{l}\text { Shashikumar et } \\
\text { al.,2017[51] }\end{array}$ & Body-Worn & $\mathrm{CNN}$ & $\begin{array}{c}\text { Atrial Fibrillation(AF) is the most widely seen } \\
\text { cardiac arrhythmia which is requires early detection } \\
\text { and monitoring to avoid potential risks. In order to } \\
\text { achieve this a CNN based framework was proposed } \\
\text { to classify AF from PPG signals. The spectrogram } \\
\text { was derived using the continuous wavelet transform } \\
\text { method. }\end{array}$ & $\begin{array}{c}\text { Self } \\
\text { [Data collected using Samsung } \\
\text { Simband] }\end{array}$ \\
\hline Liang et al.,2018[52] & Ambient & $\mathrm{CNN}$ & $\begin{array}{l}\text { They have evaluated that deep learning methods, } \\
\text { specifically the pre-trained CNN(GoogleNet) } \\
\text { outperforms the classical signal processing and } \\
\text { feature extraction methods during the classification } \\
\text { of PPG signals to estimate hypertension risk } \\
\text { stratification based on abnormal blood pressure. }\end{array}$ & $\begin{array}{c}\text { Self } \\
\text { [Multiparameter intelligent } \\
\text { monitoring in } \mathrm{ICU}]\end{array}$ \\
\hline $\begin{array}{c}\text { Ravichandran et } \\
\text { al.,[53] }\end{array}$ & Ambient & $\mathrm{CNN}$ & $\begin{array}{l}\text { Proposed end-to-end deep learning model called } \\
\text { RespNet based on CNN to extract the respiration } \\
\text { signal from PPG instead of respiration rate. They } \\
\text { tested their model on two publicly available datasets } \\
\text { named CapnoBase and Vortal. }\end{array}$ & CapnoBase and Vortal dataset \\
\hline
\end{tabular}


Table VI: Sensor-based Vital Health Monitoring using Hybrid Deep Learning Architecture

\begin{tabular}{|c|c|c|c|c|}
\hline Reference & Sensor Modality & $\begin{array}{c}\text { Deep Learning } \\
\text { Model }\end{array}$ & Description & Dataset \\
\hline Liu et al.,2018[54] & $\begin{array}{l}\text { Ambient } \\
\text { [Built-in } \\
\text { microphones of } \\
\text { smartphones ] }\end{array}$ & $\begin{array}{c}\mathrm{CNN}+ \\
\text { Bidirectional RNN }\end{array}$ & $\begin{array}{l}\text { Developed a hybrid deep model called DeepFilter } \\
\text { based on bidirectional RNN stacked with } \\
\text { convolutional layers for fine grained breathing rate } \\
\text { monitoring. The model achieved a professional } \\
\text { accuracy with experiments conducted on real-time } \\
\text { smartphone sound recording data. }\end{array}$ & $\begin{array}{c}\text { Self } \\
\text { [Breathing sound recording } \\
\text { data] }\end{array}$ \\
\hline $\begin{array}{l}\text { Gotlibovych et } \\
\text { al.,2018[55] }\end{array}$ & Body-Worn & $\begin{array}{l}\text { CNN + LSTM } \\
\text { based RNN }\end{array}$ & $\begin{array}{l}\text { Proposed a state of the art end-to-end hybrid deep } \\
\text { learning model based on CNN and RNN with LSTM } \\
\text { to detect Atrial Fibrillation from raw PPG data. }\end{array}$ & $\begin{array}{c}\text { Self } \\
\text { [PPG data from University of } \\
\text { California, San Francisco] }\end{array}$ \\
\hline Biswas et al.,2019[56] & Body-Worn & $\mathrm{CNN}+\mathrm{LSTM}$ & $\begin{array}{c}\text { Proposed a novel hybrid deep learning model based } \\
\text { on CNN and two layers of LSTM to estimate the } \\
\text { heart rate from PPG signal collected in ambulant } \\
\text { environment. }\end{array}$ & $\begin{array}{c}\text { Self }+ \text { Evaluated on TROIKA } \\
\text { dataset }\end{array}$ \\
\hline
\end{tabular}

\section{CONCLUSION}

The AAL ecosystem is influenced by a gamut of technologies which has to work in tandem to accomplish AAL tasks at various levels right from measuring the natural or man-made variable in a physical environment to the inference of results by human-being or machine. In order to leverage services delivered by any AAL environment, context awareness and seamless connectivity to the devices are of paramount importance. Some of the use cases in the AAL demand the devices to be of small form factor, less energy and memory consuming, enhanced processing and communication capabilities. The aim of AAL is to conceive the components which can be easily integrated into devices of daily use which brings down the cost involved for AAL system. Particularly, for HAR and VHSM automatic feature learning through deep learning architectures is most widely embraced by scientific community today instead of opting for handcrafted features. These deep learning architectures require high performance computing GPUs (Graphical Processing Unit) and software frameworks. To provide the insights into recent happenings regarding HAR and VHSM in research community we presented the review of papers which are categorized according to deep learning architecture (generative, discriminative or hybrid), sensor modality (body-worn/ambient/hybrid) datasets(public/self) adopted by them.

\section{REFERENCES}

1. B. Mirkin and M. B. Weinberger, "The Demography of Population Ageing," Dep. Econ. Soc. Aff. United Nations Secr., no. January, pp. 8-10, 2010.

2. U. N. Department of Social and Economic Affairs, "World Population Prospects The 2017 Revision," in World Population Prospects, 2017, pp. $11-12$.

3. National Sample Survey Office, "Elderly In India," in Ministry of Statisitcs and Programme Implementation,Govt of India, 2016, pp. 1-95.

4. R. Li, B. Lu, and K. D. McDonald-Maier, "Cognitive assisted living ambient 22 system: a survey," Digit. Commun. Networks, vol. 1, no. 4, pp. 229-252, 2015.

5. M. Memon, S. R. Wagner, C. F. Pedersen, F. H. Aysha Beevi, and F. O. 23 Hansen, "Ambient Assisted Living healthcare frameworks, platforms, standards, and quality attributes," Sensors (Switzerland), vol. 14, no. 3, pp. 4312-4341, 2014.

6. R. Chavarriaga et al., "The Opportunity challenge : A benchmark database for on-body sensor-based activity recognition," Pattern Recognit. Lett., vol. 34 , no. 15 , pp. 2033-2042, 2013.

7. T. Hayashi, M. Nishida, N. Kitaoka, and K. Takeda, "DAILY ACTIVITY RECOGNITION BASED ON DNN USING ENVIRONMENTAL SOUND," in 23rd European Signal Processing Conference (EUSIPCO),
2015 , pp. 2306-2310.

8. L. Deng, "A tutorial survey of architectures, algorithms, and applications for deep learning," APSIPA Trans. Signal Inf. Process., vol. 3, no. 2014, pp. $1-29,2014$.

9. J. Martens, I. Sutskever, and G. Hinton, "Generating Text with Recurrent Neural Networks," in Proceedings of the 28th International Conference on Machine Learning, 2011, pp. 1017-1024.

10. G. E. Hinton, "DeepBeliefNetworks," Scholarpedia, vol. 4(5), p. 5947 2009.

11. G. E. Hinton, S. Osindero, and Y.-W. Teh, “f,” Neural Comput., vol. 18, no 7, pp. 1527-1554, 2006 .

12. R. Yamashita, M. Nishio, R. Kinh, G. Do, and K. Togashi, "Convolutional neural networks: an overview and application in radiology," Insights Imaging, vol. 9, no. 4, pp. 611-629, 2018.

13. L. Zhang, X. Wu, and $\mathrm{D}$. Luo, "Real-time activity recognition on smartphones using deep neural networks," in Proceedings - 2015 IEEE 12th International Conference on Ubiquitous Intelligence and Computing, 2015 IEEE 12th International Conference on Advanced and Trusted Computing, 2015 IEEE 15th International Conference on Scalable Computing and Communications, 20, 2015, pp. 1236-1242.

14. M. A. Alsheikh, A. Selim, D. Niyato, L. Doyle, S. Lin, and H.-P. Tan, "Deep Activity Recognition Models with Triaxial Accelerometers," Artif. Intell Appl. to Assist. Technol. Smart Environ. Pap. from 2016 \{AAAI\} Work. Phoenix, Arizona, USA, vol. WS-16-01, pp. 1-8, 2016.

15. S. Bhattacharya and N. D. Lane, "From smart to deep: Robust activity recognition on smartwatches using deep learning," in 2016 IEEE International Conference on Pervasive Computing and Communication Workshops, PerCom Workshops 2016, 2016, pp. 1-6.

16. A. Wang, G. Chen, C. Shang, M. Zhang, and L. Liu, "Human Activity Recognition in a Smart Home Environment with Stacked Denoising Autoencoders," in International Conference on Web-Age Information Management, 2016, vol. 9098, pp. 29-40.

17. B. Almaslukh, A. Jalal, and A. Abdelmonim, "An Effective Deep Autoencoder Approach for Online Smartphone-Based Human Activity Recognition,” Int. J. Comput. Sci. Netw. Secur., vol. 17, no. 4, pp. 160-165, 2017.

18. M. Zeng et al., "Convolutional Neural Networks for Human Activity Recognition using Mobile Sensors," in Proceedings of the 6th International Conference on Mobile Computing, Applications and Services, 2014, vol. 6, pp. 197-205.

19. W. Jiang and Z. Yin, "Human Activity Recognition Using Wearable Sensors by Deep Convolutional Neural Networks," in Proceedings of the 23rd ACM international conference on Multimedia - MM '15, 2015, pp. 1307-1310.

20. M. Inoue, S. Inoue, and T. Nishida, "Deep recurrent neural network for mobile human activity recognition with high throughput," Artif. Life Robot., vol. 23, no. 2, pp. 173-185, 2016.

21. M. Edel and E. Köppe, "Binarized-BLSTM-RNN based Human Activity Recognition,” Int. Conf. Indoor Position. Indoor Navig. IPIN, no. October, pp. 4-7, 2016.

22. D. Ravi, C. Wong, B. Lo, and G.-Z. Yang, "A deep learning approach to on-node sensor data analytics for mobile or wearable devices," IEEE J. Biomed. Heal. Informatics, vol. 21, no. 1, pp. 56-64, 2017.

23. S.-M. Lee, "Human activity recognition from accelerometer data using Convolutional Neural Networks," in IEEE International Conference on Big Data and Smart Computing (BigComp), 2017, vol. 62, pp. 915-922.

24. F. J. O. Morales and D. Roggen, "Deep convolutional feature transfer across mobile activity recognition domains, sensor modalities and locations," Proc. 2016 ACM Int. Symp. Wearable Comput. - ISWC '16, pp. 92-99, 2016.

25. Y. Zheng, Q. Liu, E. Chen, Y. Ge, and J. L. Zhao, "Exploiting multi-channels deep convolutional neural networks for multivariate time series classification," Front.

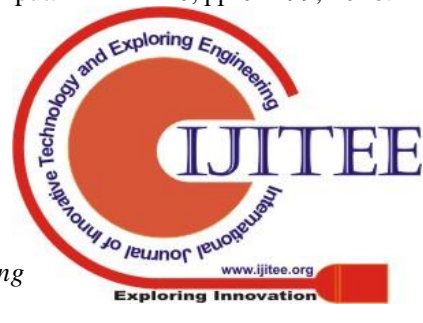




\section{Ambient Assisted Living: A Research On Human Activity Recognition And Vital Health Sign Monitoring Using Deep Learning Approaches}

Comput. Sci., vol. 10, no. 1, pp. 96-112, 2016.

26. Y. Guan and T. Ploetz, "Ensembles of Deep LSTM Learners for Activity Recognition using Wearables," IMWUT, vol. 1, no. 2, p. 11.1-11.28, 2017.

27. X. Li et al., "Concurrent Activity Recognition with Multimodal CNN-LSTM Structure," 2017.

28. M. Alzantot, S. Chakraborty, and M. Srivastava, "SenseGen: A Deep Learning Architecture for Synthetic Sensor Data Generation," in Distributed Analytics and Information Science International Technology Alliance, 2017, pp. 188-193.

29. L. Piwek, D. A. Ellis, S. Andrews, and A. Joinson, "The Rise of Consumer Health Wearables: Promises and Barriers," PLOS Med., vol. 13, no. 2, p. e1001953, 2016.

30. "ZigBee Wireless Sensor Applications for Health, Wellness and Fitness," in ZigBee Alliance, no. March, 2009.

31. Y. Khan, A. E. Ostfeld, C. M. Lochner, A. Pierre, and A. C. Arias, "Monitoring of Vital Signs with Flexible and Wearable Medical Devices," Adv. Mater., vol. 28, pp. 4373-4395, 2016.

32. F. Keita et al., "AMON: A Wearable Multiparameter Medical Monitoring and Alert System," IEEE Trans. Inf. Technol. Biomed., vol. 8, no. 4, pp. 415-427, 2004

33. S. Xu, Y. Zhang, L. Jia, and K. Mathewson, "Soft Microfluidic Assemblies of Sensors, Circuits and Radios for the Skin," Science (80-. )., vol. 344, no. 6179, pp. 70-74, 2014.

34. J. Allen, "Photoplethysmography and its application in clinical physiological measurement," IOP Sci. - Physiol. Meas., vol. 1, pp. R1-R39, 2007.

35. M. Folke, L. Cernerud, M. Ekstrom, and B. Hok, "Critical review of non-invasive respiratory monitoring in medical care," Med. Biol. Eng. Comput., vol. 41, no. 4, pp. 377-383, 2003.

36. L. Guo, L. Berglin, U. Wiklund, and H. Mattila, "Design of a garment-based sensing system for breathing monitoring," Text. Res. J., vol. 83, no. 5, pp. 499-509, 2013.

37. O. Atalay, W. R. Kennon, and E. Demirok, "Weft-knitted strain sensor for monitoring respiratory rate and its electro-mechanical modeling," IEEE Sens. J., vol. 15, no. 1, pp. 110-122, 2015.

38. G. Schwartz et al., "Flexible polymer transistors with high pressure sensitivity for application in electronic skin and health monitoring," Nat. Commun., vol. 4, no. May, pp. 1858-1859, 2013.

39. E. Yoo and S. Lee, "Glucose Biosensors: An Overview of Use in Clinical Practice," Sensors (Switzerland), vol. 10, no. 5, pp. 4558-4576, 2010.

40. D. Bruen, C. Delaney, L. Florea, and D. Diamond, "Glucose sensing for diabetes monitoring: Recent developments," Sensors (Switzerland), vol. 17, no. 8 , p. $1866,2017$.

41. V. Jindal, J. Birjandtalab, M. B. Pouyan, and M. Nourani, "An Adaptive Deep Learning Approach for PPG-Based Identification," in 38th Annual International Conference of the \{IEEE\} Engineering in Medicine and Biology Society, \{EMBC\} 2016, Orlando, FL, USA, August 16-20, 2016, 2016, pp. 6401-6404.

42. S. Lee and J. H. Chang, "Oscillometric blood pressure estimation based on deep learning," IEEE Trans. Ind. Informatics, vol. 13, no. 2, pp. 461-472, 2017.

43. W. Gu, "Non-intrusive blood glucose monitor by multi-task deep learning," in 16th ACM/IEEE International Conference on Information Processing in Sensor Networks, Pittsburgh, PA, USA, 2017, pp. 249-250.

44. X. Li, S. Wu, and L. Wang, "Blood Pressure Prediction via Recurrent Models with Contextual Layer," in Proceedings of the 26th International Conference on World Wide Web, \{WWW\} 2017, Perth, Australia, April 3-7, 2017, 2017, pp. 685-693.

45. P. Su, X.-R. Ding, Y.-T. Zhang, J. Liu, F. Miao, and N. Zhao, "Long-term Blood Pressure Prediction with Deep Recurrent Neural Networks," in 2018 IEEE EMBS International Conference on Biomedical Health Informatics, \{BHI\} 2018, Las Vegas, NV, USA, March 4-7, 2018, 2018, no. May 2017 , pp. 323-328.

46. J. Martinsson, A. Schliep, B. Eliasson, C. Meijner, S. Persson, and O. Mogren, "Automatic blood glucose prediction with confidence using recurrent neural networks," in Proceedings of the 3rd International Workshop on Knowledge Discovery in Healthcare Data co-located with the 27th International Joint Conference on Artificial Intelligence and the 23rd European Conference on Artificial Intelligence (IJCAI-ECAI, 2018), Stoc, 2018, vol. 2148, pp. 64-68.

47. Q. Sun, M. V. Jankovic, L. Bally, and S. G. Mougiakakou, "Predicting Blood Glucose with an LSTM and Bi-LSTM Based Deep Neural Network," in 2018 14th Symposium on Neural Networks and Applications (NEUREL), 20-21 November,2018, 2018, pp. 978-1-5386-6974-7.

48. Y. Cho, N. Bianchi-Berthouze, and S. J. Julier, "DeepBreath: Deep learning of breathing patterns for automatic stress recognition using low-cost thermal imaging in unconstrained settings," in 2017 7th International Conference on Affective Computing and Intelligent Interaction, ACII 2017, San Antonio, TX, USA, October 23-26, 2017, 2018, vol. 2018-Janua, pp. 456-463.

49. U. M. Khan, Z. Kabir, S. A. Hassan, and S. H. Ahmed, "A Deep Learning Framework using Passive WiFi Sensing for Respiration Monitoring," in Global Communications Conference, \{GLOBECOM\} 2017, Singapore,
December 4-8, 2017, 2017, pp. 1-6.

50.U. R. Acharya, H. Fujita, O. S. Lih, M. Adam, J. H. Tan, and C. K Chua, "Automated detection of coronary artery disease using different durations of ECG segments with convolutional neural network," Knowledge-Based Syst., vol. 132, pp. 62-71, 2017.

51.S. P. Shashikumar, A. J. Shah, Q. Li, G. D. Clifford, and S. Nemati, "A deep learning approach to monitoring and detecting atrial fibrillation using wearable technology," in 2017 IEEE EMBS International Conference on Biomedical and Health Informatics, BHI 2017, Orland, FL, USA, February 16-19, 2017, 2017, pp 141-144.

52.Y. Liang, Z. Chen, and R. Ward, "Photoplethysmography and Deep Learning: Enhancing Hypertension Risk Stratification,' Biosensors, vol. 8, no. 4, pp. 1-13, 2018.

53.V. Ravichandran et al., "RespNet: A deep learning model for extraction of respiration from photoplethysmogram," Comput. Res. Repos., vol. abs/1902.0, pp. 1-4, 2019.

54.B. Liu et al., "Deep Learning versus Professional Healthcare Equipment: A Fine-Grained Breathing Rate Monitoring Model," Mob. Inf. Syst., pp. 1-9, 2018.

55.Gotlibovych et al., "End-to-end Deep Learning from Raw Sensor Data: Atrial Fibrillation Detection using Wearables," Comput. Res. Repos., vol. abs/1807.1, 2019

56.D. Biswas et al., "CorNET: Deep Learning framework for PPG based Heart Rate Estimation and Biometric Identification in Ambulant Environment," IEEE Trans. Biomed. Circuits Syst., pp $1-11,2019$. 